Meta

Journal des traducteurs

Translators' Journal

\title{
La traducción del francés al español (lengua segunda terminal) en una perspectiva textual
}

\section{Ricardo Serrano}

Volume 33, numéro 2, juin 1988

L'enseignement de la traduction au Canada - Teaching Translation in Canada

URI : https://id.erudit.org/iderudit/004092ar

DOI : https://doi.org/10.7202/004092ar

Aller au sommaire du numéro

Éditeur(s)

Les Presses de l'Université de Montréal

ISSN

0026-0452 (imprimé)

1492-1421 (numérique)

Découvrir la revue

Citer cet article

Serrano, R. (1988). La traducción del francés al español (lengua segunda terminal) en una perspectiva textual. Meta, 33(2), 283-298.

https://doi.org/10.7202/004092ar d'utilisation que vous pouvez consulter en ligne. 


\section{LA TRADUCCION DEL FRANCES AL ESPANOL (LENGUA SEGUNDA TERMINAL) EN UNA PERSPECTIVA TEXTUAL}

RICARDO SERRANO

Université du Québec à Trois-Rivières, Québec

Con una función diferente pero un poco a la vieja manera de la Escuela de Traductores de Toledo, el castellano viene a ser la tercera lengua de traducción en el Baccalauréat en Traduction impartido por el Département de langues modernes de l'Université du Québec à Trois-Rivières.

Ello implica un planteamiento de la enseñanza de la traducción relativamente novedoso, abierto a una tercera lengua y condiciona, por otra parte, la orientación general del conjunto de los cursos de la tercera lengua, cuya progresión culmina precisamente en la traducción.

Hay que notar que el bloque de cursos de español sirve una amplia gama de motivaciones entre las que detectamos especialmente la cooperación interuniversitaria, en pleno desarrollo con los países de lengua española (y que corresponde sin duda, además, a una necesidad primaria en une pequeña universidad como la nuestra).

Este medio de enseñanza a que aludimos nos sitúa de entrada fuera de las prácticas de traducción más trilladas y tratadas en nuestro contexto geográfico : inglésespañol-inglés (en USA) y francés-inglés-francés (en Canadá), terrenos en los que se han generado habitualmente los análisis teóricos que han trazado las grandes coordenadas de nuestra disciplina.

Por otra parte, el curso a que principalmente nos referiremos, Traduction français-espagnol, desarrollado en la sesión de Invierno 85, representa el término del actual bloque de cursos de español pero sus alumnos presentan todavía ciertas necesidades de perfeccionamiento en lengua española.

Recuérdese la función pedagógica atribuida tradicionalmente a la traducción dentro de la enseñanza de las lenguas segundas y de la perspectiva humanista, linea de la que seguimos siendo deudores (un tanto híbridos, en esta integracíon nuestra de mercado de trabajo y enseñanza superior).

Ante esta situación-cajón de sastre, identificada por una triple necesidad (continuación del aprendizaje de la lengua española, solución de problemas de traducción francés-español, consideración de ciertos aspectos teóricos ligados al fenómeno de la traducción), determinamos dos objetivos prioritarios, ligados a sendos conjuntos de contenidoslactividades :

1. Matizar los "niveles" de la lengua española, que es, en nuestro contexto, lengua segunda terminal (L2T) en el proceso de traducción. 


$\begin{array}{ll}\text { Acercamientos } & \text { semántico } \\ & \text { sociolingüístico } \\ \text { (fonéticolortológico) } & \text { etimológico } \\ & \text { (ortográfico) }\end{array}$

2. Sensibilizar a los estudiantes hacia la necesidad de un análisis textual implícito a la práctica de traducción : reivindicación de un planteamiento textual de la traducción, en el que centraremos este trabajo.

Pero, ante todo, ilustraremos rápidamente los grandes tipos de actividades que han rodeado en nuestra experiencia pedagógica el primer objetivo de diversificación de niveles:

- Consideraciones de campo semántico (series "no semétricas ») :

Pasar de la serie " matin - midi - après-midi — soir " a " mañana - mediodía — tarde » (con toda la enormidad de diferencias sociológicas implícitas).

De la serie "bois-forêt» a "bosque-(forestal)-selva» [más el residuo "madera-leña »].

- Consideraciones de nivel de lengua-comunicación :

La ramificación léxica entre un nivel culto|científico|técnico|profesional y un nivel coloquial|patrimonial (hilo/filamento, orejeras/auriculares, etc.).

Marcas de respeto (quiere...); de pertenencialadhesión (movida); de situación (cuarto de baño/servicios).

Implicaciones fonéticas y ortológicas (la pronunciación de la $\mathrm{d}$ final en "Madrid », o de la -d- intervocálica).

Implicaciones etimológicas de la ortografía : la $\mathbf{h}$ (f) de hilo.

- Trasformaciones de sinonimia con aplicaciones directas a L2T (para evitar repeticiones).

- Oposiciones-clarificaciones semánticas siguiendo el hilo de polisemia, hominimia, cuasi-hominimia (índece/indicio y, más lúdicamente, rata/gata/nata/lata/etc.).

- En cuanto a los textos utilizados en las prácticas de traducción, hemos tratado igualmente de diferenciar al máximo el nivel de lengua, el contenido y la situación :

- Un artículo de Paul BUSSIERES sobre la "biomasa » aparecido en uno de los útimos números de Interface (ACFAS).

- Un artículo reciente de Pierre FOGLIA (la Presse) sobre homosexualidad, buen ejemplo del estilo coloquial que caracteriza el autor.

- Dos fragmentos de una versión francesa del Lazarillo de Tormes con anotaciones terminológicas.

- Varias series de frases programadas sobre problemas específicos (uso de pronombres, posición de los adverbios, etc.).

- Un fragmento de una publicación gubernamental, le Québec dans le monde (1984), que hablada precisamente de las relaciones con países de Hispanaoamérica. d'été ".

- Una historieta escrita y dibujada por Enric SIO y publicada en Pilote : «Solstice

- Un fragmento de un cuento para ninõs, la historia de dos conejos en una granja mecanizada : l'île aux lapins, de J. MULLER, J. STEINER.

Con estos dos últimos textos llegamos, efectivamente (entre el lenguaje infantil o juvenil, el papel de la imagen y el hecho de traducir a L2), al planteamiento de verdaderos problemas-límite de la traductibilidad.

Estos problemas nos conducen a una verdadera crítica (casi recomposición) de los dos siguientes textos, seleccionados precisamente en ese terreno propicio : 
- Un fragmento de F. NIETZSCHE, de Ainsi parlait Zarathoustra.

- Un fragmento de G. DELEUZE, "Pensée nomade".

Objetivo : poner a los traductores en ciernes ante los límites del aforismo, del retruécano, de lo decible|comunicable que fuerzan un primer intento de comprensión y de análisis del texto, fuera desde el principio de la concepción de la traducción como paso de un clisé, de una forma sentencial y cerrada a otra equivalente en LT.

¿Cómo dar, si no, ese dificil salto, tan a menudo quiástico, de la traducción (piénsese en los fenómenos de desplazamiento de orden).

¿Cómo conservar un dinamismo temático a través de un proceso que atraviesa enormes diferencias de neutralización|oposición ? (piénsese, por ejemplo, en las diferencias de función de tiempos verbales entre francés y español).

¿Cómo desarrollar la sensibilidad y la intuición del traductor ?. ?Qué hilo conductor y qué bases metodológicas ofrecerle?

Todas estas preguntas nos conducen a la afirmació de la necesidad de un planteamiento textual (supraoracional) de la traducción, a un análisis textual previolimplícito a nuestra práctica.

MODELO PROPUESTO PARA UN ANÁLISIS TEXTUAL ORIENTADO A LA TRADUCCIÓON

Nótese ante todo que la función pedagógica de este esquema no es tanto un permanente y riguroso seguimiento como un despertar de la sensibilidad textual en la práctica de la traducción. Consideramos, por tanto, que la "duplicación del trabajo escrito" (de la que algún estudiante se me ha quejado) puede quedar esencialmente limitada al periodo de formación como tal y que es " exportable " y aplicable, en tanto que herramienta adquirida, al trabajo así como a otras eventuales aplicaciones futuras.

En cuanto a sus bases teóricas, enraízan en la línea formalismo-estructuralismosemiología y siguen concretamente algunos intentos (Coquet, Veron) de formalización de la "lógica del lenguaje natural".

Intentamos movernos en este terreno, actualmente en pleno desbroce, con un criterio de "rigor práctico " (al que no es extraño el " comentario de textos " de LazaroCorrea), que nos conduce a un cuerpo flexible de convenciones formales fundamentalmente representables en clase de manera visual, gráfica y locativa.

Procedemos además con una doble orientación sincrética y económica (metodológicamente) que nos lleva a considerar la integración de ciertos modelos de análisis de la narración nacidos en el campo de la etno|sociolingüística (Labov), aplicables a menudo con éxito a textos de nivel coloquial.

\section{EL METODO}

1. Fase : Lectura-Subrayado

Comprender el significado del texto y, para ello :

- Subrayar lo importante (con un rotulador bien visible)

- Señalar (con lápiz) las palabras no conocidas

- Buscar en el diccionario las palabras no conocidas (y anotar el sentido en el mismo texto cuando sea posible).

\section{Fase : Presentacion}

- Señalar si es un texto independiente o un fragmento

- Determinar el tipo de escrito : descripción, narración, argumentación, (diálogo)

- Señalar, eventualmente, el género literario al que pertenece de manera concreta 
- Dar indicaciones precisas pertinentes.

3. Fase : «Logo»

Realizar un esquema visual del contenido del texto utilizando el conjunto de símbolos reseñados a continuación :

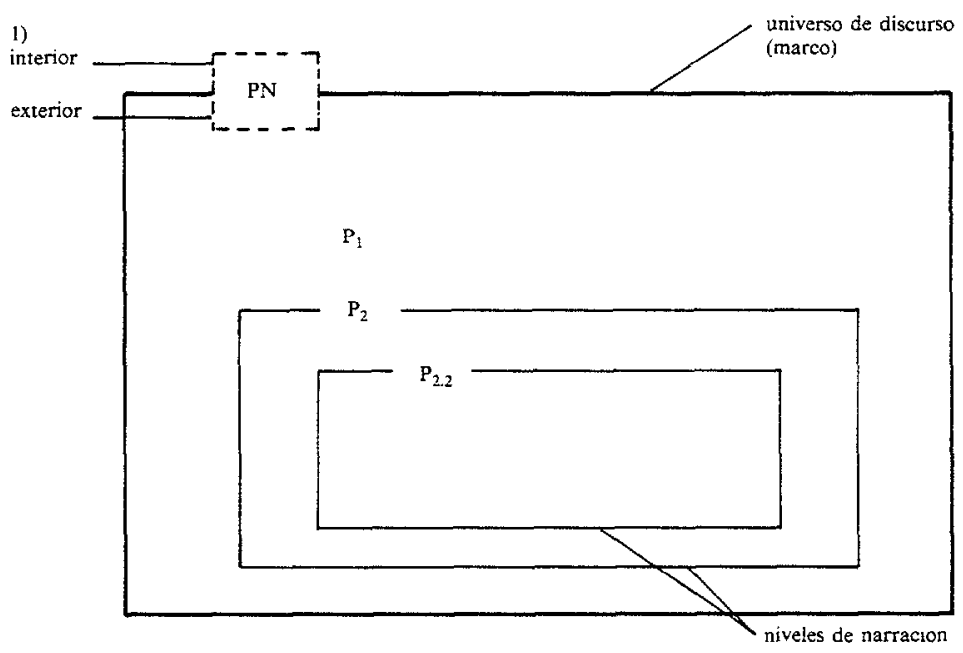

$\mathrm{PN}=$ Personaje narrador, personaje que habla. Es un « lugar » (topos) que crea el autor para, desde él, "ver", generar el discurso.

EL PN es así el punto de vista, el "polo » del discurso del texto y de su universo.

Uno de los atributos de este "marco" es la verosimilitud (o presuposición, desde otro punto de vista), la adecuación de un texto a su medio y a su contexto, aquello que es concebible o esperable, lo narrable (donde se sitúan tanto la " convención " como la " literalidad").

EL PN puede ser

- interior : si también actúalactuó en el mundo que representa el texto (forma autobiográfica)

- exterior : si sólo habla.

Cana uno de los personajes puede, a su vez, convertirse en un PN (sistema de cajas chinas de Las mil y una noches).

\section{RELACIONES PN - P}

Visión de los personajes:

- "desde fuera " : visión exterior objetiva (típica de la novela policiaca). PN sabe sólo hechos y comportamientos.

- "con" (desde dentro) : PN sabe lo mismo que P, lo que piensa o quiere.

- "por encima »: PN sabe más que P, (lo que va a pasar, por ejemplo).

Actitud de la visión de los personajes :

- $\mathbf{P}$ inferior (monigote)

- P igual - libertad + necesidad, abstracción,

- P superior (héroe) deformación de P

Relaciones especiales :

- Proyección : relación privilegiada PN-P que se materializa a menudo en forma de visión "por encima "/ con». 
P central : aquel que es objeto de una proyección.

- P Filtro : el P central a través de cuya psicología vemos al resto de los personajes.

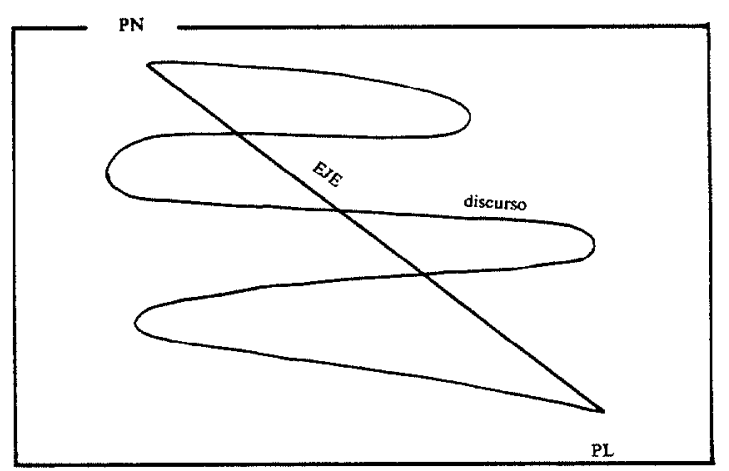

DEL CONCEPTO DE PN AL DE PERSONAJE LECTOR (PL)

Si PN es uno de los polos del texto, el otro es PL, el personaje lector, anticipación del lector real implícita en el mismo texto.

Al igual que PN, PL puede ser interior (cuando está presente en forma de tú, vosotros, Ud., etc.) o exterior (cuando PN no se refiere expresamente a un "Tú " explícito).

En cuanto a su representación en estos esquemas, sólo incluiremos a PL INT cuando así sea necesario.

PN y PL constituyen los dos puntos definidores de la Linea-eje alrededor de la que se desarrolla el discurso y que constituye la ideología del texto.

PL, como PN, puede aparecer más o menos delimitado en un tiempo y en un lugar así como en una concreta posición ideológica. Podemos suponer que estas delimitaciones actúan en el mismo sentido y en igual medida para PN y PL pero el paso del tiempo sobre un texto, entre otros factores, puede desplazar a este último hacia una posición de rebeldía con respecto al contenido.

3) LA ESTRUCTURA LOGICA FUNDAMENTAL (en la que privilegiamos, frente a otros acercamientos reseñados por Vazquez-Ayora, la relación de contradicción)

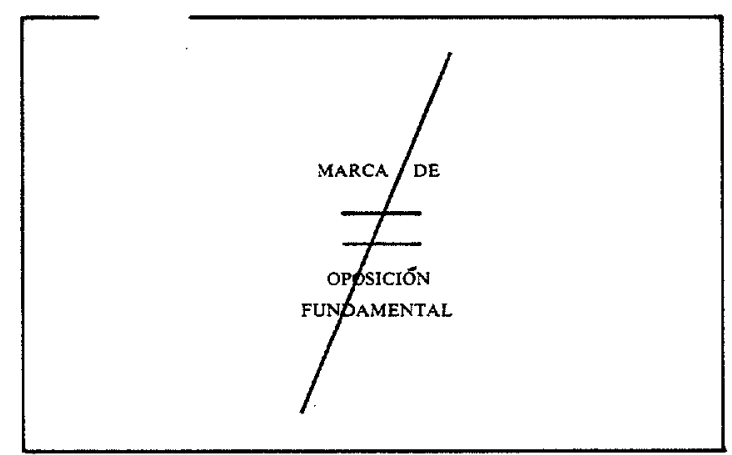


4) LA ESTRUCTURA TEMPORAL (que se integra, como segundo principio, con la anterior)

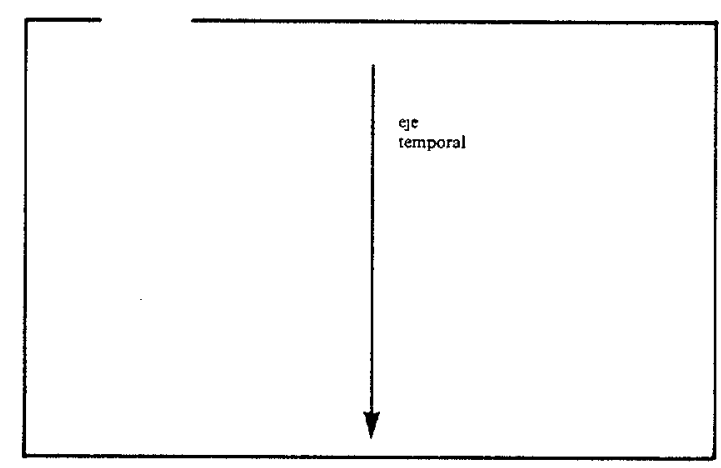

5) LOS OPERADORES SECUNDARIOS (a los que se podrían añadir una serie de elementos de modulación) :

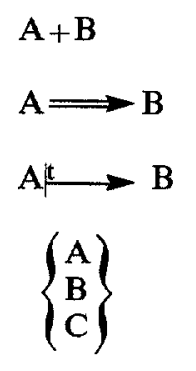

(A)

Esquemas

jerárquicos, de estructura

familiar, etc.

$$
\begin{aligned}
& \mathrm{T}_{1} \\
& \mathrm{~T}_{2}
\end{aligned}
$$

$\mathbf{A}=\mathbf{B}$

$-\mathbf{A}$

$A \neq B$

A

$A \in B /\left(\begin{array}{l}B \\ A\end{array}\right)$ suma (serial)

implicación

transformación

Alternativa

facultativamente

organigrama

ordenación

temporal

igualdad

negación

oposición

eliminación

pertenencia

(a una clase)
A y B

si A, entonces B

A se trasforma en B

$A \circ B \circ C$

A puede estar o no

A, B, y C están organizados así

Tiempo 1, tiempo 2

A es igual a B

No $A$

Máxima : si A, -B Mínima : A y B son diferentes

A deja de contar

$A$ es $\mathbf{B}$ 


\section{Fase : Resumen-Argumento}

Un resumen es una representación sintética del "discurso ", del o del texto

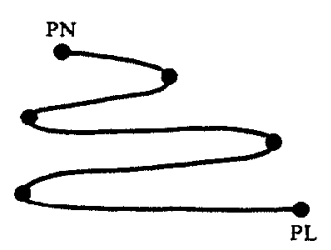

Es el mismo texto más corto.

Conserva el orden del texto.

Conserva el PN del texto y su forma (salvo en el caso de un texto dialogado, que se reduce a narración, y en el caso de un $\mathrm{PN}$ interior, que se convierte en $\mathrm{PN}$ exterior).

En el caso espefico de la narración de carácter coloquial, podemos distinguir además los siguientes tipos de elementos (importando a Labov) :

\subsubsection{PRESENTACION}

Responde a la pregunta ¿De qué se trata?

... A mí me han operado también de apendicitis

\subsubsection{ORIENTACION (IDENTIFICACION)}

Responde a las preguntas ¿Quién, ¿Qué ?, ¿Cuándo ?, ¿Dónde ?

Estaba en casa, cuando...

\subsubsection{DESARROLLO (HECHOS).}

Responde a la preguna ¿Qué pasó ? <... Vino el médico > y ...

Formas de secuenciación

- encastre : historia dentro de la historia

- concatenación : serie de secuencias

- alternancia : entre prop. de una primera secuencia y una segunda

\subsubsection{EVALUATION}

Responde a la pregunta hipotética ?pero, por qué me cuentas eso ? Muestra la razón de haber contado el hecho (le da importancia)

${ }_{i}$ Fue terrible!

\subsubsection{RESULTADO}

Responde a la pregunta ?qué fué de todo ello?

Por fin, entré en el quirófano y me operaron

\subsubsection{CODA}

Coincide parcialmente con el resultado y consiste en

- señal de fin

- observaciones generales 
- vuelta del tiempo pasado de los hechos al tiempo presente

Después ya no he vuelto a tener más complicaciones

\section{Fase : Tema}

Todo texto « discurre " por un " terreno" : el tema (tomamos este término en un claro sentido textual, no sentencial)

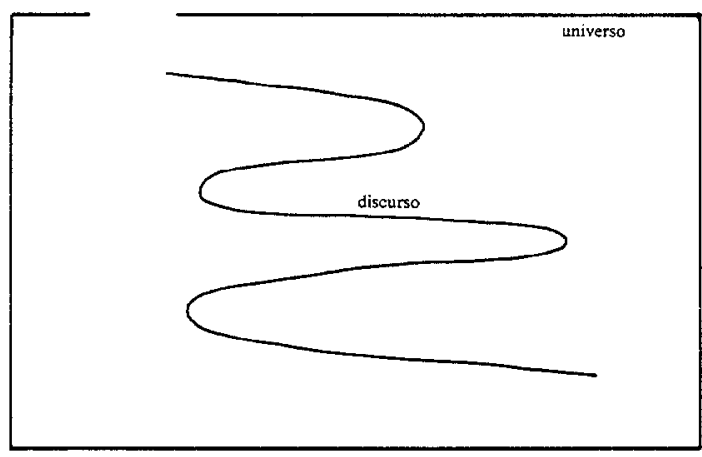

El tema es el "universo del discurso".

El tema es lo conocido/conocible sobre el asunto (v. notas sobre $\mathrm{PN}=$ límite del universo del discurso).

El tema es no sólo lo que dice el texto sino, eventualmente, lo que no dice. del texto.

La presentación del tema tiene su propio orden, lógico independiente del orden

El «PN del tèma »se identifica con el propio analista.

Ahora bien, ?nuestra determinación del tema, en cuanto analistas, debe seguir la dirección ideológica del texto (1) o superarla (2)?

Si (1), tema = ideología.

Si (2), posición que defendemos, tema > ideología, donde esta queda identificada con el eje del discurso.

\section{Fase : Apartados}

El tema muestra primordialmente una relación lógica.

La articulación textual de esta relación lógica produce apartados, que muestran serialmente cada uno de los elementos de la relación central.

El trabajo de esta fase consiste pues en desglosar la idea unitaria del tema en una serie de elementos integrantes que corresponden a fragmentos del texto o, lo que es 10 mismo, hacer la relación entre el resultado de la fase 4 (resumen) y el de la fase 5 (tema).

\section{Fase : Forma Expresiva}

Dentro de cada apartado, realizaremos una búsqueda minuciosa para determinar todas les " expresiones significativas " en las que hemos « leído " (muchas veces intuitivamente hasta aquí) el tema o sus elementos. y forma.

A partir de este momento, logramos una verdadera « lectura en relieve » de fondo 
8. Fase : Localizacion

- Determinar

de enunciación

de enunciado

- Localizar el texto dentro de la obra

- Aportar datos pertinentes sobre

- estructura político-social

- tendencias del arte y la cultura del tiempo

- vida del autor

\section{Fase : Conclusion}

- Balance sintético de las fase anteriores.

- Opinión personal razonada y concreta acerca del texto.

\section{UNA APLICACION DE CLASE}

El el contexto descrito al principio de este artículo y durante las dos premeras semanas de febrero 85 , hacemos, tras algún intento anterior más limitado, una primera aplicación de conjunto con preparación previa individual y discusión colectiva en clase. El texto seleccionado para esta experiencia es un fragmento del artículo ya mencionado de Pierre FOGLIA, Masculins singuliers, aparecido tan sólo unos días antes.

Reproducimos a continuacín dicho fragmento, que ocupa un lugar central en el conjunto del texto :

Comment dire ?... Prenons les intellectuels hétéros qui ont toujours été les alliés naturels des minorités opprimées. Or, depuis quelques années, comme par hasard depuis que les homos s'affichent plus librement, il est devenu très " in" dans les milieux traditionnellement libéraux, gens de lettres entre autres, de se déclarer "anti-tap ". Comprenez anti-tapettes. On entend couramment : "C'est assez-là, c'est devenue une vraie mafia ! Ils (les taps) occupent tout l'espace culturel, au théâtre il n'y en a que pour eux, la littérature est devenue le territoire presque exclusif des lesbiennes, en musique ce sont les pédés qui nous ont imposé le disco... ", etc., etc. Subtil détour ! Le préjugé se trouve ici une excuse économico-culturelle : une soi-disant mafia... Être homosexsuel, entre deux insultes, je préférerais la moins sournoise, me faire carrément insulter par le premier brave imbécile venu, plutôt que me faire reprocher de prendre trop de place par quelque joyeux lettré qui n'oserait jamais me dire "sale tapette", parce que c'est pas cool. Même si au fond c'est exactement ce qu'il pense...

Comment dire ? ... Ce matin, j'étais à Saint-Hyacinthe chez la mère d'un homosexuel. Une vraie " dame de la rue Panet " comme on écrivait jadis dans les journaux pour désigner la ménagère la plus " ordinaire " qui soit. Une dame qui, avant de découvrir que son fils était homosexsuel, ne savait de l'homosexsualité que les clichés les plus gros, les plus bas, les plus gras. Elle m'a raconté le chemin qu'elle a fait avec son fils, et je suis sorti de chez elle quasiment les larmes aux yeux. Ça faisait longtemps que j'avais entendu une aussi belle histoire d'amour...

Resumen del resultado de la discusión de clase en las fases de trabajo más determinantes

FASE 2 (PRESENTACION)

- Fragmento central de un artículo de periódico reciente

- Exposición|argumentación

- Asunto candente (homosexualidad)

- Estilo coloquial, personalización 
FASE 3 ("LOGO ")

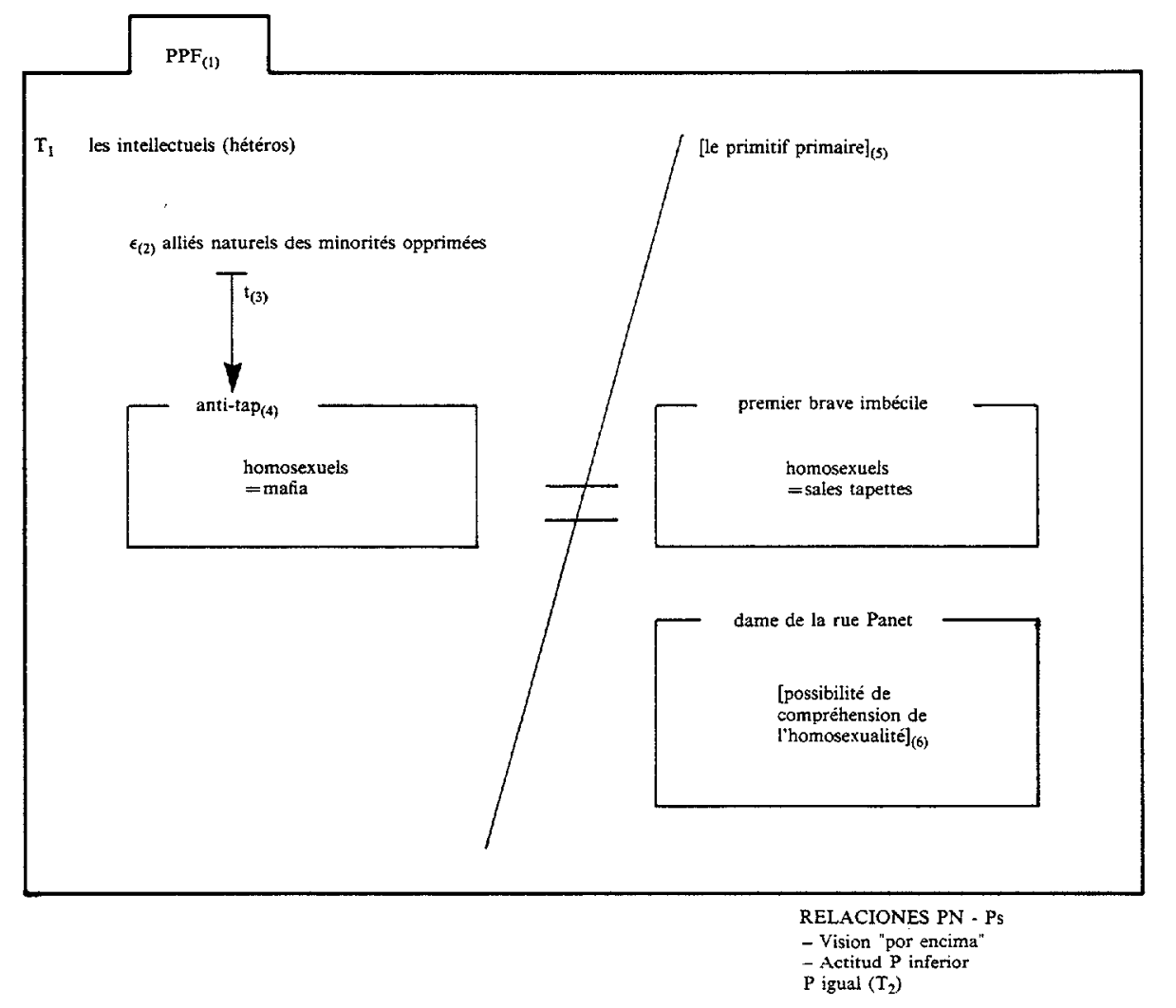

NOTAS AL "LOGO":

1. PPF $=$ «Personaje Pierre Foglia » (si se nos permite distinguir al autor del PN) : entre snob y (buena) gente (de la calle).

2. : Podría utilizarse, si se prefiere, el signo $=$.

3. Nótese que esta trasformación, que supone una evolución inscrita en un tiempo histórico, representa también una oposición secondaria.

4. Se trata, naturalmente, de los intelectuales (desenmascarados), que producen el discurso representado en la caja correspondiente.

5. En oposición a "intellectuels», proyectamos una expresión genérica cuya aparición en el texto queda impedida por la inundación de la imaginería de "premier brave imbécile".

6. En la misma línea de nuestra nota anterior, objetivamos el discurso de la señora, tan sólo aludido. Nótese, además, que la actitud de la señora gana a PN (y a PL).

\section{FASE 4 (RESUMEN)}

Ante la afirmación cultural de los artistas homosexuales, los intelectuales pasan de ser aliados naturales suyos a considerarles como una mafia molesta, excusa retorcida peor que el insulto primario. prensión.

Porque a partir del insulto primario la gente de la calle sí puede llegar a la com- 
FASE 5 (TEMA), donde pretendemos reflejar más un diálogo de clase que un resultado cerrado :

La homosexualidad es todavía hoy, efectivamente, un tabú y el homosexual, un (grupo) marginado.

¿Cuáles son los mecanismos ideológicos de la marginación ?. ¿Por qué y cómo una diferencia se convierte en un elemento de marginación ? ¿Cuáles son los caminos de afirmación de un grupo marginado ? ¿Qué trasformación de la sociedad puede implicar la afirmación pública de un grupo marginado como los homosexuales ? ¿Grupo o individuos, por cierto?

¿Por qué esos componedores de ciencia e ideología que son los intelectuales, los escritores, parecen cerrarse en banda contra esa supuesta invasión del espacio del arte y la literatura por parte de los homosexuales?

¿Representan realmente los homosexuales una nueva sensibilidad ? ?Representan realmente los grupos marginales la potencialidad de un nuevo discurso ?

¿Es la actitud de los intelectuales personificados en el texto un comportamiento de tipo corporatista, una defensa de un terreno considerado como propio y en el que se ven pisados?

¿No sale toda esta historia un poco demasiado cuadrada con esa "dame de la rue Panet " que parace simpática pero cuyo discurso desconocemos?

[Téngase en cuenta que este tipo de encaminamiento hacia la determinación del tema, acerca de la que nos referíamos anteriormente a la alternativa entre limitarse a las posiciones ideológicas del texto o excederlas, representa sin duda una problemática fundamental para el traductor, ese ser anfibio que tiene que escribir para otra sociedad, pasar a otros moldes, conjugar (entre su texto y sus notas de traductor) los originales PN y $\mathrm{PL}$ con los nuevos que de hecho él está creando].

FASE 7 (FORMA EXPRESIVA), a la que saltamos :

¿Por qué, de hecho, nos quedamos sin enterarnos de la historia de la señora ? (tras el fragmento elegido, no se vuelve ya a saber de ella).

Todo el texto está atravesado de anafóricas marcas de inefabilidad : "Comment dire... ". ?Son generadas estas marcas por el rechazo social (PL) de la homosexualidad, por un ambiente de trabajo entre hostil y "blague anti-tap" donde el texto se ha producido, por la falta de un discurso-« otro" (a pesar de la supuesta invasión de la que se hace mención) ? : en cualquier caso, son limitaciones, carencias significadas/significativas, afasias, imposibilidades, inefabilidades.

Pregunta hipotética, recursiva justificación además de este artículo, pero de necesario planteamiento en un contexto de enseñanza universitaria : ¿Y cómo traduciríamos estas marcas hacia una lengua|sociedad donde esas limitaciones no existieran ? Respuesta : o bien señalando en nota el carácter de les marcas, si se decide mantenerlas en el texto $\mathrm{T}$; o bien, si se decidiera eliminarlas, mencionando, en nota también, su existencia y significado en el texto $O$.

\section{Traducción-resultado del texto propuesto a la luz de los elementos de juicio antteriores.}

Cómo les diría..., tomemos el caso de nuestros liberales intelectuales [ "tradicionalmente " desaparece para no alargar más el sintagma nominal ; "nuestros " lo cubre, así como a " heterosexuales" que algún estudiante propone], que han sido siempre aliados naturales de las minorías oprimidas pero que, desde hace algunos años, como por casualidad, desde que los homosexuales se presentan más libremente a la luz del día, comienzan a tomar posiciones contra ellos : "Esta gente se ha convertido en una verdadera mafia ! Están monopolizando todo el espacio cultural : no hay ya más teatro que el suyo, la literatura es el territorio casi exclusivo de las lesbianas [" tortilleras », si nos de- 
jamos arrastrar por los matices más coloquiales del texto O], la música " disco " nos la han impuesto los maricones... ». Sutil deslizamiento : el prejuicio encuentra aquí una excusa económico-cultural, una supuesta mafia... Pero de ser homosexual [si yo fuera homosexual + marca 1) persona], estoy por asegurarles que, entre dos insultos, preferiría el menos retorcido de cualquier imbécil primario al reproche de ocupar demasidado sitio venido de un tipo muy leído y muy escribido que no se atrevería nunca a llamarme "maricón" porque eso no es muy elegante, aunque sea exactamente lo que está pensando.

Cómo les diría... [,miren, + insistencia fática] esta misma mañana estaba [he estado + marca de 1) persona] en Saint-Hyacinthe en casa de la madre de un homosexual, un ama de casa corriente y moliente que, antes de descubrir que su hijo lo era, no conocía sobre la homosexualidad más que algunos clisés groseros. Esta mujer me ha contado el camino que ha recorrido y, al final, he salido de su casa con lágrimas en los ojos : hace mucho tiempo que nadie me había contado una historia de amor como esa.

\section{ALGUNAS REFERENCIAS BIBLIOGRAFICAS}

Reseñamos a continuación las obras citadas en el presente artículo o tenidas en cuenta directamente para su redacción.

BUNGE, Mario (1980) : Epistemología, Barcelona, Ariel.

COQUET, Jean-Claude (1974) : "Sémantique du discours et analyse de contenu ", Connexions, no 11.

ECO, Umberto (1976) : Tratado de semiótica general (Edición española de $A$ Theory of Semiotics.

GARCIA YEBRA, Valentín (1982) : Teoría y práctica de la traducción, Madrid, Gredos.

LABOV, W. (1972) : Language in the Inner City, Univ. Pennsylvania Press.

LAZARO, F, E. CORREA $\left(1967^{5}\right)$ : Cómo se comenta un texto literario, Salamanca, Anaya.

OTERO, Carlos-Peregrin (1970) : Instroducción a la lingüistica transformacional, México, Siglo XXI.

POLO, José (1972) : Lingüistica, investigación y enseñanza, Madrid, OEI.

POLO, José (1976) : El español como lengua extranjera, enseñanza de idiomas y traducción, Madrid, SGEL. STEEL, Brian (1983) : Ejercicios de traducción del español. Nivel superior, Madrid, EDI-6.

VAZQUEZ-AYORA, Gerardo (1977) : Introducción a la traductología, Washington, Georgetown University Press.

VERON, Eliseo (1973) : "Linguistique et sociologie. Vers une 'logique naturelle des mondes sociaux' ", Communications, $\mathrm{n}^{\circ} 20$. 